\title{
Spontaneous Coronary Artery Dissection and Anomalous Coronary Origin - Underlying Cause of Acute Coronary Syndrome in a Young Woman: A Case Report
}

\author{
Roxana-Daiana Lazar, Abigaela Rus, Cosmin Tolescu, Renata Gerculy, Diana Opincariu, \\ Imre Benedek
}

Center of Advanced Research in Multimodality Cardiac Imaging, Cardio Med Medical Center, Târgu Mureș, Romania

\section{ABSTRACT}

Introduction: Spontaneous coronary artery dissection (SCAD) represents a very rare and poorly understood condition that is gaining recognition as an important cause of myocardial infarction, especially among young women. The pathogenesis of SCAD is not well established yet, but several theories have been proposed. Case presentation: We report the case of a 25-year-old woman without any history of cardiovascular disease who presented with acute anterior ST-elevation myocardial infarction (STEMI) due to the luminal obstruction generated by an intramural hematoma from a SCAD of the left main coronary artery, which was successfully treated by coronary artery stenting. Additionally, the patient presented anomalies of coronary origins (ACO) with separate emergences of the left anterior descending (LAD) artery from the left coronary cusp and the left circumflex artery (LCX) from the right coronary cusp, with no apparent clinical significance. Conclusion: SCAD should always be included in the differential diagnosis of young patients presenting with STEMI. In case of prompt diagnosis, SCAD-STEMI patients are successfully treated with percutaneous coronary intervention (PCI). Moreover, it is of vital importance to identify variants of ACO, even without clinical relevance at the moment of the acute event, in order to initiate an appropriate management, since ACO increases the risk of routine PCI.

Keywords: spontaneous coronary artery dissection, acute anterior ST-elevation myocardial infarction, anomalies of coronary origins, percutaneous coronary intervention

\section{ARTICLE HISTORY}

Received: September 5, 2020 Accepted: November 15, 2020 Published online December 16, 2020

\section{CORRESPONDENCE}

\section{Abigaela Rus}

Str. 22 Decembrie 1989 nr. 76

540124 Târgu Mureș, Romania

Tel: +40265217333

E-mail: abigaelarus@yahoo.com 


\section{INTRODUCTION}

Acute myocardial infarction and sudden cardiac death can be evoked by spontaneous coronary artery dissection (SCAD), a very rare condition which occurs predominantly in women during or after pregnancy. Even though several mechanisms have been hypothesized, no etiology throws light upon the pathogenesis of SCAD. Additionally, anomalous coronary origin (ACO) is a considerable but rather infrequent cause of sudden cardiac death among young individuals, while aberrant origin of the left circumflex artery (LCX) from the right coronary cusp is extremely exceptional. The two underlying conditions could both individually lead to acute coronary events, but so far there were no studies revealing any associations between them.

SCAD causes myocardial ischemia and myocardial infarction (MI) due to obstruction of coronary blood flow from intimal dissection and/or intramural hematoma formation, ${ }^{1}$ while the mechanism through which SCAD develops seems to be divided into two theories. On the one hand, the primary pathological event is considered to be a disruption in the vessel wall, leading to the formation of intramural hematoma. ${ }^{2}$ On the other hand, the primary event is believed to be a spontaneous hemorrhage originating from the vasa vasorum within the wall, leading to a microhematoma between the media and adventitia, eventually followed by coronary artery dissection. ${ }^{3}$ Also, wall motion abnormalities (such as hypokinesis or akinesis) were linked to the dissected arteries. ${ }^{4}$

Furthermore, ST-elevation myocardial infarction (STEMI)-SCAD plays an important part in the STEMI subset, being characterized by significantly higher frequency of left main or left anterior descending culprit and cardiogenic shock than STEMI caused by atherosclerosis, while also correlating with an overall higher incidence of inhospital mortality. ${ }^{5}$

The differential diagnosis of acute coronary syndromes (ACS) caused by SCAD includes atherosclerotic ACS, coronary artery spasm, Takotsubo cardiomyopathy (brokenheart syndrome), coronary embolism, and myocardial infarction with non-obstructed coronary arteries. ${ }^{6}$ Emergency coronary angiography along with intravascular ultrasonography (IVUS) or optical coherence tomography

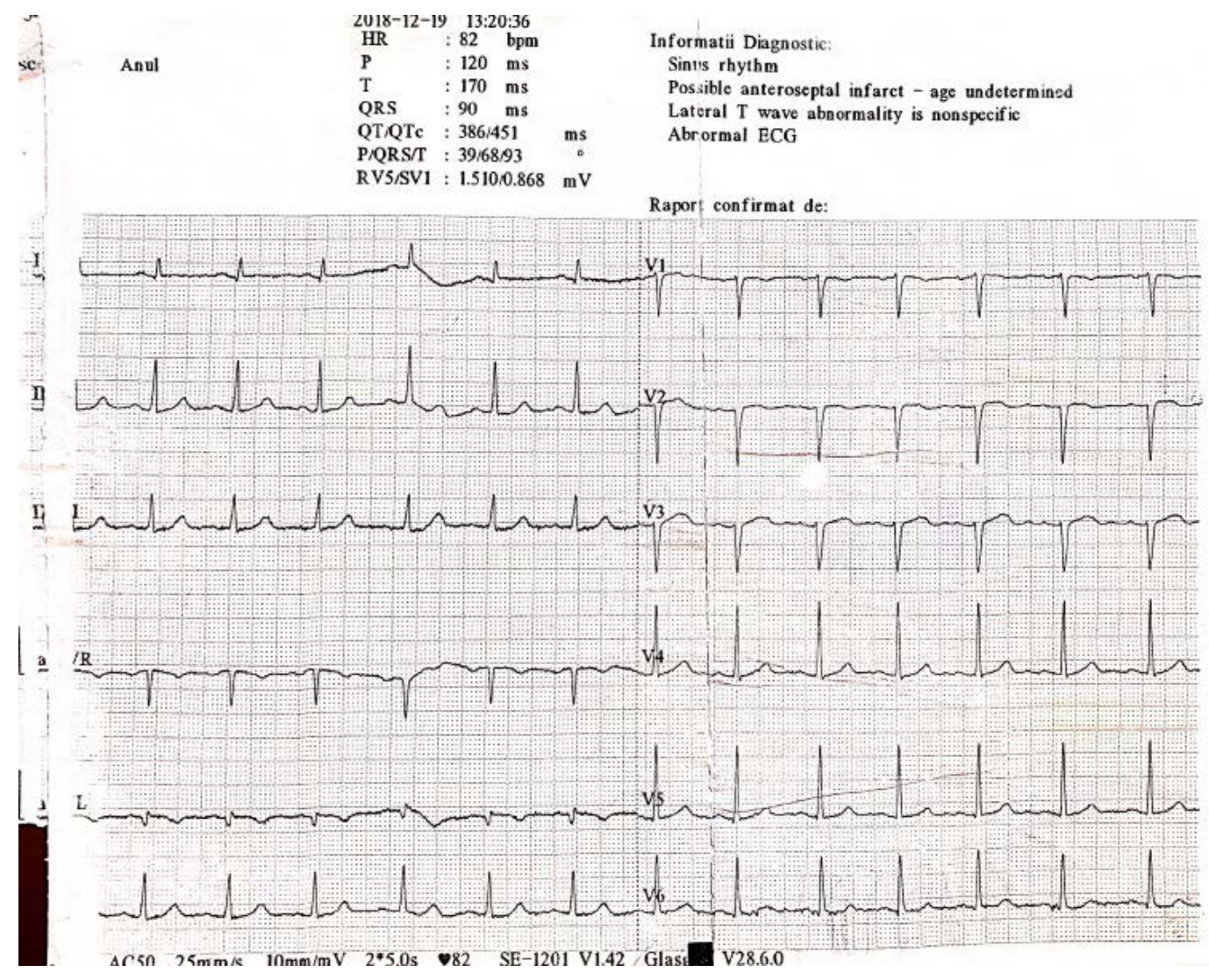

FIGURE 1. Minor ST-segment elevations in $\mathrm{aVL}, \mathrm{V}_{1}$ to $\mathrm{V}_{3}$ 


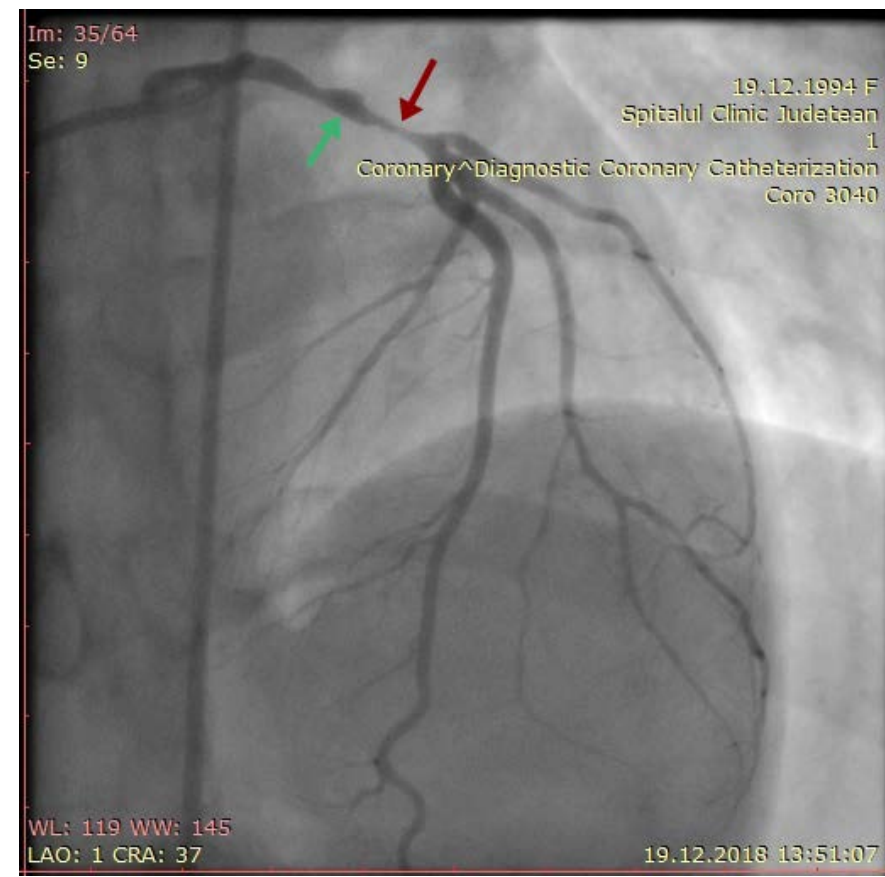

FIGURE 2. Left coronary angiography revealing a $90 \%$ tubular stenosis of the left main (red arrow), considered to be caused by an intramural hematoma, with proximal aneurysmal enlargement (green arrow)
(OCT) are key investigations in guiding the diagnosis towards SCAD.

The therapeutic strategy of ACS due to SCAD should be decided individually for each patient and includes conservative treatment, percutaneous coronary intervention (PCI), or coronary artery bypass graft (CABG) surgery.

\section{CASE PRESENTATION}

We report the case of a 25-year-old woman without any history of cardiovascular disease, brought to the emergency department after developing severe chest pain with onset 10 hours before admission and extended duration over 3 hours, which ceased after administration of nitroglycerin. On presentation, the patient's blood pressure was 120/80 mmHg, heart rate was 77 beats/min, respiratory rate was 15 breaths/min, and oxygen saturation was above $98 \%$ on ambient air. No relevant family history of cardiovascular disease was exposed from the medical history of the patient. On admission the patient presented with mild hypokalemia $(3.4 \mathrm{mmol} / \mathrm{L})$, the white blood cell count was $8,790 / \mathrm{mm}^{3}$, hemoglobin $12.5 \mathrm{~g} / \mathrm{dL}$, platelet

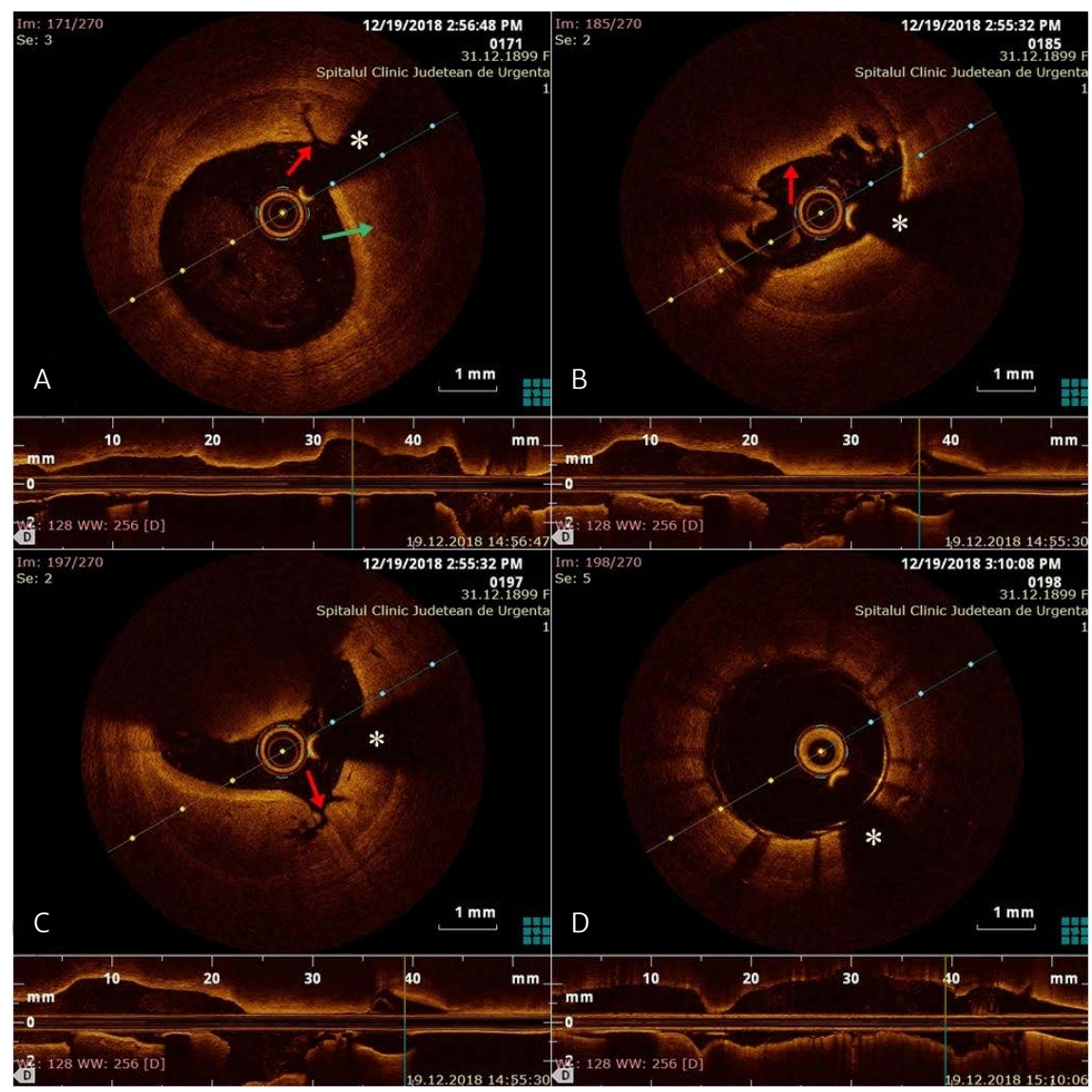

FIGURE 3. OCT confirmed intimal tear with intramural hematoma in the proximal segment of the LMCA (green arrow), indicating also the intimal rupture (red arrow) 


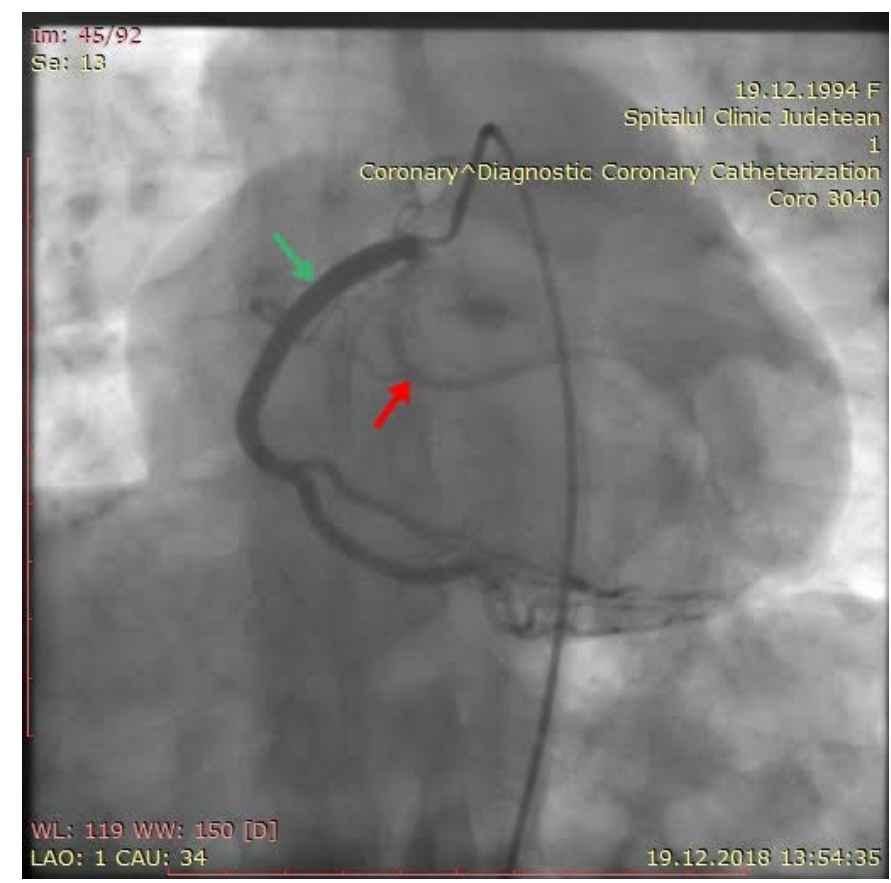

FIGURE 4. Right coronary angiography indicating origin of the left circumflex artery from the right coronary cusp (red arrow), together with the right coronary artery (green arrow)

count 272,000/mm $\mathrm{mm}^{3}$, total cholesterol $152 \mathrm{mg} / \mathrm{dL}$, triglyceride $105 \mathrm{mg} / \mathrm{dL}$, creatine kinase myocardial brain (CKMB) $143 \mathrm{ng} / \mathrm{mL}$, and serum high-sensitivity troponin-I $7,732 \mathrm{ng} / \mathrm{mL}$.

Electrocardiography revealed sinus rhythm with anterolateral ST-segment elevations in aVL, V1 to V3 (Figure 1). Transthoracic echocardiogram (TTE) revealed an efficient ejection fraction of 50\%, hypokinesis of apex and apical segment of interventricular septum. Therein, clinical presentation combined with laboratory examination, 12-lead ECG, and TTE vouched for acute anterior STEMI.

Therefore, emergency coronary angiography was performed, which uncovered a $90 \%$ stenosis from an extended tubular lesion over $1 \mathrm{~cm}$ (considered to be due to intramural hematoma) with aneurysmal enlargement at mid segment of the left main coronary artery (LMCA) (Figure 2). The possibility of iatrogenic coronary dissection was ruled out in this case. OCT confirmed intimal tear with intramural hematoma on the proximal segment of the LMCA, which pointed out the underlying cause of luminal obstruction (Figure 3). Accordingly, a drug-eluting stent was implanted in the LMCA, without involvement of the bifurcation of the LMCA in the left anterior descending artery (LAD) and ramus intermedius artery (confirmed by post-procedural OCT), via percutaneous transluminal coronary angioplasty. After successful PCI, TIMI flow 3 was established.
Additionally, right coronary angiography demonstrated ACO with separate emergences of the LAD from the left coronary cusp and the LCX from the right coronary cusp (Figure 4). The other coronary artery segments did not present any significant angiographic abnormalities.

The patient recovered uneventfully and is doing well without any further complications until present time. Also, she did not experience any episodes of angina pectoris, and she endured physical exertion satisfactorily until present days.

The publication of the case was agreed by the patient and approved by the ethics committee of the institution.

\section{DISCUSSION}

SCAD correlates with an overall higher incidence of inhospital mortality. From the same perspective, PCI was linked to a higher mortality in SCAD patients presenting with non-ST-segment elevation myocardial infarction than in those presenting with STEMI. ${ }^{5}$ Accordingly, it is documented that the last subset of patients benefit from primary PCI (leading to successful results in most STEMISCAD patients), with low 3-year mortality. ${ }^{7}$ In case of single-vessel disease with short segment involvement, PCI is recommended but it should be performed with caution due to an increased likelihood of false lumen or propagating the dissection distally. ${ }^{8}$

Among the risk factors for SCAD, studies have described non-coronary vasculopathy, especially fibromuscular dysplasia (FMD), pregnancy or postpartum state, extreme stressors, emotions or exercise, collagen disorders, systemic inflammatory conditions. ${ }^{1}$ Also, the disease has a higher prevalence among women with severe hypertension, cocaine abuse, and use of oral contraceptives. ${ }^{8}$ None of the above were found in our patient, her only risk factor was her female gender.

Furthermore, in patients presenting with ACS in whom the diagnosis of SCAD is not certain, coronary artery tortuosity (four times more common in women with SCAD) or other angiographic features (coronary microaneurysms, FMD) could be useful as markers of SCAD. ${ }^{9}$

Although in this case the anomalous origin of the LCX does not seem to have any clinical relevance on its own, it is crucial to also identify each variants and ACO, as in some cases they can generate drastic reduction of blood flow to the myocardium (ischemia) and cause chest pain, arrhythmias, and sudden cardiac death, while also increasing the risk of routine procedures (surgery or angioplasty). ${ }^{10}$ Early diagnosis and immediate initiation of suitable treatment are usually linked to favorable outcome. 
The particularity of this case lies within the absence of risk factors and association with ACO, which remains unclear until the present moment, due to lack of data. What is certain is that both conditions can individually lead to acute coronary events and must not be overlooked. Even though patients with left main coronary artery dissection have a mortality of $50 \%,{ }^{8}$ our patient benefited from PCI with direct stenting and recovered uneventfully 20 months after the event.

\section{CONCLUSION}

As shown in multiple studies, SCAD stands for a complex entity, with considerable implications in terms of clinical approach and diagnosis; hence, it should always be included in the differential diagnosis of young patients presenting with ACS, especially women in the peripartum state. Clinicians should also pay attention to the coronary abnormalities which may transcend to potentially severe cardiac events, since recognition of these coronary abnormalities is the key for a suitable therapeutic management.

\section{CONFLICT OF INTEREST}

Nothing to declare.

\section{ACKNOWLEDGEMENT}

This research was supported via the research grant no. 103544/2016, contract number 26/01.09.2016, entitled "Increasing the research capacity in the field of vulnerable plaque imaging, based on advanced nanoparticles, fusion imaging and computational simulation - PlaqueImage", financed by the Romanian Ministry of European Funds, the Romanian Government and the European Union.

\section{REFERENCES}

1. Tweet MS, Gulati R, Hayes SN. What Clinicians Should Know About Spontaneous Coronary Artery Dissection. Mayo Clin Proc. 2015;90:1125-1130. doi: 10.1016/j.mayocp.2015.05.010.

2. Hayes SN, Kim ESH, Saw J, et al. Spontaneous Coronary Artery Dissection: Current State of the Science: A Scientific Statement From the American Heart Association. Circulation. 2018;137:e523-e557. doi: 10.1161/CIR.0000000000000564.

3. Kwon TG, Gulati R, Matsuzawa Y, et al. Proliferation of Coronary Adventitial Vasa Vasorum in Patients With Spontaneous Coronary Artery Dissection. JACC Cardiovasc Imaging. 2016;9:891-892. doi: 10.1016/j.jcmg.2015.11.030.

4. Saw J, Mancini GB, Humphries K, et al. Angiographic appearance of spontaneous coronary artery dissection with intramural hematoma proven on intracoronary imaging. Catheter Cardiovasc Interv. 2016;87:E54-E61. doi: 10.1002/ ccd.26022.

5. Mahmoud AN, Taduru SS, Mentias A, et al. Trends of Incidence, Clinical Presentation, and In-Hospital Mortality Among Women With Acute Myocardial Infarction With or Without Spontaneous Coronary Artery Dissection: A Population-Based Analysis. JACC Cardiovasc Interv. 2018;11:80-90. doi: 10.1016/j. jcin.2017.08.016.

6. Adlam D, Alfonso F, Maas A, Vrints C; Writing Committee. European Society of Cardiology, acute cardiovascular care association, SCAD study group: a position paper on spontaneous coronary artery dissection. Eur Heart J. 2018;39:3353-3368. doi: 10.1093/eurheartj/ehyo80.

7. Lobo AS, Cantu SM, Sharkey SW, et al. Revascularization in Patients With Spontaneous Coronary Artery Dissection and ST-Segment Elevation Myocardial Infarction. J Am Coll Cardiol. 2019;74:1290-1300. doi: 10.1016/j.jacc.2019.06.065.

8. Akyuz A, Alpsoy S, Akkoyun DC. Spontaneous coronary artery dissection and woven coronary artery: three cases and a review of the literature. Korean Circ J. 2013;43:411-415. doi: 10.4070/kcj.2013.43.6.411.

9. Eleid MF, Guddeti RR, Tweet MS, et al. Coronary artery tortuosity in spontaneous coronary artery dissection: angiographic characteristics and clinical implications. Circ Cardiovasc Interv. 2014;7:656-662. doi: 10.1161/ CIRCINTERVENTIONS.114.001676.

10. Villa AD, Sammut E, Nair A, Rajani R, Bonamini R, Chiribiri A. Coronary artery anomalies overview: The normal and the abnormal. World J Radiol. 2016;8:537-555. doi: 10.4329/wjr. v8.i6.537. 